\title{
Escala sobre atitudes da aprendizagem colaborativa associada às tecnologias da informação e comunicação: Validade e diferenças nas IES públicas e privadas na cidade de Natal-RN
}

Scale on collaborative learning attitudes associated with information and communication technologies: Validity in public and private universities in Natal-RN

Escala de actitudes de aprendizaje colaborativo asociadas a las tecnologías de la información y la comunicación: Validez y diferencias en IES públicas y privadas de la ciudad de Natal-RN

Recebido: 09/03/2021 | Revisado: 16/03/2021 | Aceito: 18/03/2021 | Publicado: 24/03/2021

\author{
Jorge Assef Lutif Júnior \\ ORCID: https://orcid.org/0000-0002-3058-6116 \\ Universidade Potiguar, Brasil \\ E-mail: lutifjr@gmail.com \\ Antonio Jorge Fernandes \\ ORCID: https://orcid.org/0000-0003-0145-017X \\ Universidade Potiguar, Brasil \\ Universidade de Aveiro, Portugal \\ E-mail: afer@ua.pt \\ Nilton S. Formiga \\ ORCID: https://orcid.org/0000-0003-4907-9736 \\ Universidade Potiguar, Brasil \\ E-mail: nsformiga@yahoo.com
}

\begin{abstract}
Resumo
O principal objetivo deste artigo é demonstrar a percepção do aluno quanto à aplicação de TICs em sala de aula como mecanismo colaborativo de aperfeiçoar e inovar o rito de aprendizagem. Com base nos dados a serem obtidos, será efetuado uma análise comparativa qualitativa a fim de identificar grupos que possuam características similares sobre a percepção do uso das TICs e sistemas de avaliação adotados. Participaram do estudo 228 universitários, todos eles da cidade de Natal-RN, sendo o maior percentual etário na faixa de idade de 22 a 29 anos, bem como, a maioria eram de graduação, com uma maior concentração de respondentes no início do curso de Ciências Contábeis e a maioria de IES privada. Estes responderam, além de dados sociodemográficos e socio-acadêmicos, também, a escala de atitudes frente as TICs. Com base nos resultados, os indicadores psicométricos das medidas utilizadas, sugerem que as escalas são confiáveis, bem como, apresentam grande potencial de análise diagnóstica de gestão das TICs em acadêmicos em universidades públicas e privadas. Em termos gerais, os resultados destacam a necessidade de uma maior atenção para o tipo de curso e o período em que os universitários se encontram, pois, acredita-se ser uma condição adequada para aplicar as TICs.
\end{abstract}

Palavras-chave: Ensino; Tecnologias educacionais; Metodologias ativas; Web educação.

\begin{abstract}
The main objective of this dissertation is to demonstrate the student's perception regarding the application of ICTs in the classroom as a collaborative mechanism to improve and innovate the learning rite. Based on the data to be obtained, a qualitative comparative analysis will be carried out in order to identify groups that have similar characteristics on the perception of the use of ICTs and assessment systems adopted. A total of 228 university students participated in the study, all of whom were from the city of Natal-RN, with a higher age percentage in the age group of 22 to 29 years, as well as the majority were undergraduates, with a greater concentration of respondents at the beginning of the Science course. Accounting and most private universities. These responded, in addition to sociodemographic and socio-academic data, also the scale of attitudes towards ICTs. Based on the results, the psychometric indicators of the measures used, suggest that the scales are reliable, as well as have great potential for diagnostic analysis of ICT management in academics at public and private universities. In general terms, the results highlight the need for greater attention to the type of course and the period in which the university students are, since it is believed to be an adequate condition for applying ICTs.
\end{abstract}

Keywords: Teaching; Educational technologies; Active methodologies; Web education. 


\section{Resumen}

El objetivo principal de este artículo es demostrar la percepción del alumno sobre la aplicación de las TIC en el aula como mecanismo colaborativo para mejorar e innovar el rito de aprendizaje. A partir de los datos que se obtengan, se realizará un análisis cualitativo comparativo con el fin de identificar grupos que tengan características similares sobre la percepción del uso de las TIC y los sistemas de evaluación adoptados. En el estudio participaron un total de 228 estudiantes universitarios, todos ellos de la ciudad de Natal-RN, con mayor porcentaje de edad entre los 22 y 29 años, así como, la mayoría de ellos eran estudiantes de pregrado, con una mayor concentración de encuestados. al inicio del curso Ciencias Contables y la mayoría de IES privadas. Estos respondieron, además de los datos sociodemográficos y socioacadémicos, también la escala de actitudes hacia las TIC. Con base en los resultados, los indicadores psicométricos de las medidas utilizadas, sugieren que las escalas son confiables, además de tener un gran potencial para el análisis diagnóstico de la gestión de las TIC en académicos de universidades públicas y privadas. En términos generales, los resultados resaltan la necesidad de una mayor atención al tipo de curso y al período en el que se encuentran los estudiantes universitarios, ya que se considera una condición adecuada para la aplicación de las TIC.

Palabras clave: Ensenãnza; Tecnologías educativas; Metodologías activas; Educación web.

\section{Introdução}

Considerando as concepções teóricas destacadas por alguns autores tanto no contexto brasileiro quanto em outros países, é possível observar nas produções, um desenvolvimento de importantes elementos conceituais e práticos, referente as novas perspectivas metodológicas na relação ensino-aprendizagem, as quais, centradas na definição e aplicabilidades das tecnologias de informação e comunicação (TIC's) destinadas ao processo educacional (Maia, 2003; Corrêa, 2005; Pozo, 2008; Vieira, 2011; Karsenti, 2012; Mendonça, 2013; Blau; Shamir-Inbal,2017).

De acordo com Figueiredo (2009) O fato de ter as TIC's em destaque nas IES, não é algo que ocorre no vazio sócio educacional, mas, surge, a partir das recentes demandas, implementações e formatações do uso de tecnologias em sala de aula, seja via sistemas computacionais ou via aparelhos de comunicação móvel. Em especial, é preciso salientar, que a influência dessa conjuntura, deve-se ao surgimento e manipulação dos smartphones na modalidade de ensino superior (IES), os quais, destinados aos cursos das mais variadas áreas de formação profissional (Vilaça; Araújo, 2016)

De acordo com Almekhlafi, Ismail e Al-Mekhlafy (2017) a conexão das TICs no processo de aprendizagem em uma instituição de ensino superior é estimulada por uma transformação de paradigma no ensino que estabeleça limites no uso da caneta e quadro branco, permitindo-se em abrir um canal de comunicação capaz de dialogar com conteúdo digital inovador.

Segundo Muro e Gabriel (2016), a integração no currículo com uso da tecnologia da informação e do conhecimento baseado em metodologias ativas e associados e autonomia do aluno tornou-se uma preocupação global e é de grande importância para alcançar os objetivos de desenvolvimento do milênio e os objetivos da educação para todos da União das Nações Unidas pela Educação e Cultura - (Unesco, 2015).

De acordo com Oliveira (2020) e Pereira (2020) a relação entre tecnologia e Educação ainda é um tema bastante polêmico e envolve diferentes posições e argumentações. Constata-se que muitos ambientes educacionais proíbem o uso de celulares, smartphones, tablets, laptops e similares em sala de aula, proibição está considerada inadequada por grande parcela dos estudantes. Porém, entre os professores se verifica uma clara divisão de posicionamento, na medida em que alguns defendem o uso destes recursos e inclusive buscam ampliar as suas possibilidades de aplicação nos ambientes educacionais, enquanto outros acreditam que estas tecnologias tendem a tirar a concentração do aluno, prejudicando a sua aprendizagem.

Já Jiao (2013) e Hu (2014), trazem em suas pesquisas que tal condição constrói uma boa atmosfera emocional para atrair nos alunos a atenção e o interesse, já que o ensino por meio de sistemas tecnológicos promove a reforma do método de aprendizagem em sala de aula e exige que os professores dêem ao máximo o seu papel de liderar e de mobilizar totalmente a aprendizagem focado nos alunos, gerando assim, melhores iniciativas e entusiasmos por partes destes. Os alunos incorporam totalmente o status do assunto e objetivam realmente tornarem-se mestres da aprendizagem. 
O resultado é que os estudantes não só têm acesso a quantidades aparentemente intermináveis de informação, mas também personalizam conteúdo e reorganizam-no de uma forma que lhes permita compreender melhor um tópico e compartilhá-lo com colegas (Valente, 2013).

\section{Metodologia}

\section{Amostra}

A metodologia compreende o percurso adotado pela pesquisa, na escolha do enfoque para atender aos objetivos propostos, e da mesma maneira, realizar as preferências de quais estratégias e procedimentos foram assegurados para a validação de todo o processo. Assim, o percurso metodológico foi alinhado com o objetivo geral desse estudo que teve como foco elaborar uma medida sobre atitudes que os alunos indicam para a gestão e aplicação de TICs em sala de aula como mecanismo de autonomia e inovação para o processo de aprendizagem.

A pesquisa possui abordagem multimétodo, que incide no uso conjugado de métodos quantitativos e qualitativos para alcançar os objetivos de um mesmo estudo (Creswell, 2013).

Desse modo, o estudo empírico está composto por duas fases. A primeira fase é de caráter quantitativo e consistiu na aplicação de um questionário estruturado junto uma amostra intencional e não probabilística composta por 228 estudantes de IES localizada no município de Natal do estado do Rio Grande do Norte, matriculados nos cursos associados a gestão como administração, ciências contábeis, engenharias dentre outros. No que se refere ao critério de exclusão, considerou-se participantes que: não estavam regulamente matriculados nos cursos, não residiam no Rio Grande do Norte, ser menor de 18 anos e negar colaborar no preenchimento do questionário- sem filhos e; - residentes fora do estado da Bahia. Considerou-se ainda que, quando consultado, in loco, o participante aceitasse responder o instrumento completo.

\section{Instrumentos}

Em um primeiro momento, foi aplicada a versão online via google forms do "Questionário sobre o uso das tecnologias da informação e comunicação em sala de aula" (Silva \& Coll, 2015). No presente estudo, a análise dos dados se restringe à verificação da frequência relativa do uso das TICs pelos estudantes e da importância atribuída às atividades mediadas pelas TICs para o aprendizado colaborativo, a inovação e a criatividade dentro de sala de aula na percepção dos estudantes.

Ressaltamos que diversos estudos apontam o uso das TICs para a coleta de dados como um importante recurso de inovação metodológica na pesquisa qualitativa (Pain, 2009). A análise dos dados foi realizada com base no enfoque interpretativo (Willig, 2013).

A segunda seção irá examinar as práticas atuais e parâmetros de integração de TIC em sala de aula. O estudo utilizou de questionários da Likert na escala de 5 (cinco) pontos para coletar dados sobre a percepção dos alunos e docentes no uso das TICs em sala de aula sobre diversas concepções relacionados à: habilidades de integração, preparação, competências, inovação e autonomia (classificadas como: 1. Discordo totalmente; 2. Discordo parcialmente; 3. Indiferente; 4. Concordo parcialmente; e 5.Concordo totalmente).

Os dados coletados foram medidos a partir da percepção dos alunos no ensino superior associados às práticas atuais do uso de TICs em sala de aula. A escala de cinco pontos da Likert se deu através de questionários associado a uma coleta de dados sobre a capacidade dos alunos em aprender em sala de aula com integração de tecnologia. Para análise dos dados, foi utilizado o software estatístico SPSS relacionando as correlações existentes entre os objetivos e as questões. A análise dos dados foi obtida através de tabelas de frequência para apresentar variáveis em números e porcentagens. 
No questionário foram inclusos direcionamentos centrais tomados como base e ajustados a partir dos estudos internacionais de Karsenti, Collin e Harper-Merrett (2012), Barrette (2017) em um contexto sala de aula tecnológica.

A fundamentação deste trabalho terá como base: (a) levantamento bibliográfico; (b) aplicação de questionários aos alunos do ensino superior e (c) análise dos dados obtidos que permitam estimular a compreensão dos fatos e resultados apresentados

\section{Procedimentos e aplicação do instrumento}

Os autores do estudo, com experiência prévia na administração do instrumento foram responsáveis pela coleta dos dados in loco. Seguiu-se todos os procedimentos previsto na Resolução 466/2012 do Conselho Nacional de Saúde (CNS) e na Resolução 016/2000 do Conselho Federal de Psicologia brasileiro para as pesquisas com seres humanos e Associação Nacional de Pesquisa e Pós-Graduação em Psicologia (ANPEPP), 2000). Os instrumentos da pesquisa foram aplicados aos estudantes individualmente, através do link, o qual, hospedava o questionário eletrônico, sendo divulgados a eles por email e redes sociais.

Estes, eram convidados a participar da pesquisa acessando o link divulgado, podendo responder de acordo com a disponibilidade do tempo e interesse do participante que de forma voluntária, anônima e privada passava a preencher o questionário. Foi solicitada uma autorização prévia dirigida aos mesmos, expressa no termo de compromisso livre e esclarecido - TCLE, no qual apresentavam-lhes os objetivos da referida pesquisa.

\section{Análise de dados}

Após a coleta e tendo os dados agrupados e salvos em planilha eletrônica no programa de estatística SPSS para Windows (versão 24.0), foram analisados além do teste de normalidade amostral, uma estatística descritiva e análise do Alfa de Cronbach, correlações de Pearson, teste t de Student, análise fatorial exploratória dos principais componentes e Anova

No que se refere à análise fatorial exploratória, foram considerados alguns itens com o propósito de confirmar, ajustar os fatores relevantes e auxiliar na interpretação dos dados, seguindo as teorias propostas por Malhotras (2011), Hair, Anderson, Tatham e Black (2008). São eles: Apha de Conbrach, comunalidade, KMO, rotação Varimax, teste de esfericidade de Bartlett, média e desvio padrão.

Assim, O KMO (um dos principais itens citados) tem como propósito estatístico, mensurar a adequação da análise fatorial, na qual valores entre 0,70 e 1,0 considera a análise apropriada. Já valores abaixo de 0,70 sinalizam análise fatorial inadequada.

O KMO avalia o fenômeno provocado por uma determinada correlação entre pares de variáveis que são influenciados por outras variáveis. Em pequenos valores significam que não podem ser explicadas por outra, já acima de 0,70 significa o desejável (Hair; Anderson; Tatham; Black, 2008).

Posteriormente, o teste de esfericidade de Bartlett foi utilizado para mensurar e avaliar a hipótese de que as variáveis podem se correlacionar com a amostra coletada. Sendo resultado com um valor $\geq 0 \leq 1$, mas, também, sendo acima de 0,70 , o que significa que o conjunto de variáveis poderá ser considerada uma inter-relação destinada a análise fatorial.

Salienta-se, ainda, que o procedimento da análise fatorial contempla a análise de rotação Varimax, a qual trata-se de um método capaz de minimizar o número de variáveis com grau alto de um fator, ampliando, portanto, o conhecimento que contribui para melhorias na interpretação dos fatores.

Hair et al (2009) afirmam que o método Varimax é consideravelmente relevante em comparação a outros métodos, uma vez que apresenta critérios eficientes para conseguir uma estrutura fatorial simplificada. 
Em síntese, a análise fatorial se inicia a partir da necessidade de um estudo proveniente do problema de pesquisa. Sua técnica tem por principal objetivo resumir ou condensar as informações envolvidas em diversas variáveis de modo a facilitar a compreensão e definir os constructos importantes para explicação do fenômeno além das dimensões assumidas e características das variáveis originais (Hair; Anderson; Tatham; Black, 2008).

Além dessas análises, foram realizadas teste t de Student, correlação de Pearson, estatística descritivas (médio, desvio padrão, mediana), bem como, o qui-quadrado destinada à avaliação das frequências entre as variáveis.

\section{Resultados}

Ao terminar a coleta de dados, a qual, ocorreu por meio eletrônico, os participantes, por sua vez, tiveram suas respostas inseridas automaticamente, no programa Microsoft Excel, de acordo com o que era apresentado nos instrumentos a pesquisa respondidos no link divulgado; em seguida, as informações do Excel foram transferidas para pacote estatístico SPSS 24.0, no qual, são realizadas as análises estatísticas.

O interesse em verificar essas etapas para a pesquisa deste artigo, deve-se às seguintes direções: 1 - trata-se de uma pesquisa pioneira, tanto em sua medida quanto ao objetivo pretendido, 2 - nas recentes buscas nos sites da produção científica brasileira na área gestão escolar e gestão acadêmica do ensino superior (por exemplo, scielo.br, newpsi.bvs-psi.org.br, pepsic.bvsalud.org, www.periodicos.capes.gov.br) não foram encontrados estudos similares quanto ao tema proposto neste artigo que esteja associado ao ensino superior, escopo principal da proposta.

Sendo assim, numa primeira etapa de conferência dos resultados, buscou-se verificar se não teriam respostas duplicadas e/ou missing (itens sem respostas), condição a qual, identificada sem nenhum erro no preenchimento da planilha por parte dos respondentes.

A partir dessa avaliação inicial, verificou-se a qualidade e adequabilidade da amostra para essa pesquisa. Com isso, em relação a multicolinearidade entre as variáveis, observaram-se correlações que estiveram dentro dos parâmetros definidos por Tabachnick e Fidell (2001) [ $r \leq 0,90$, variando de 0,36 a 0,63]. Condições estas, que revelam a não existência de variáveis com alta correlação, permitindo elaborar modelos correlacionais com baixo erro de medida.

Foi avaliado também, a presença de outliers multivariados, o qual, através do teste de normalidade de KolmogorovSmirnov (KS), destinado a análise de amostras superiores a 100 sujeitos (MIOT, 2017), observou-se uma normalidade (KS = $0,79)$ da amostra a um $\mathrm{p}<0,48$.

No que diz respeito à variância comum do método (VCM) (common method variance), no presente artigo, tanto as variáveis independentes (VI) quanto as dependentes (VD) mensuram opiniões dos mesmos respondentes, podendo representar um problema de medida por similaridade; para tal avaliação, tomou-se como referência a proposta empírica de Podsakoff, MacKenzie e Podsakoff (2003), os quais, sugerem para a verificação do VCM, o teste de um fator de Harman nos dados coletados, devido atender quando critério empírico, a existência de um percentual menor do que 50\%.

Neste sentido, foi encontrado um único fator com autovalor acima de 1,00, explicando uma variância abaixo do que critério exigido pelos autores supracitados (38,67\% da covariância nas variáveis), implicando a não existência de um problema de variância comum do método.

Testou-se também, a tendência de não resposta. A partir do procedimento indicado por Armstrong e Overton (1977), foi avaliado a diferença entre os primeiros $(\mathrm{n} 1=10)$ e os últimos $(\mathrm{n} 2=10)$ participantes da pesquisa. Para isso, utilizou-se o teste de Levene e o teste $t$ para avaliar, respectivamente, a igualdade das variâncias e dos meios entre os escores médios.

Observou-se que estas condições estatísticas não foram significativas $(p<0,28)$ entre os escores médios dos constructos, bem como, o t de Student foi $\leq 1,96$ (isto é, $\mathrm{t} \leq 0,89$ ), condição a qual, revela que o viés de não resposta não será um problema significativo para as análises. 
Confirmado a qualidade da amostra, procurou-se seguir para avaliação dos objetivos principais da pesquisa; para isso, algumas etapas avaliativas, estabelecidas como hierarquia qualitativa para a construção e definição do instrumento proposto, foram seguidas, considerando o tipo de estudo.

Mas, antes de seguir para essas etapas, apresentar-se-ão as informações sócio demográficas da amostra coletada. Foram coletadas duas amostras, a primeira com 25 respondentes destinados a verificação da validade de conteúdo e segunda amostra, com 228 respondentes, destinados para avaliação da análise fatorial exploratória.

Todos os respondentes, de ambas as amostras tinha as mesmas condições demográficas: Todos eram estudantes universitário da cidade de Natal-RN, tendo maior percentual etário (36\%) na faixa de idade de 22 a 29 anos, 49\% eram de graduação, com uma maior concentração de respondentes no início do curso (68\%) e a maioria (475) do curso de Ciências Contábeis, com $68 \%$ deles de IES privada.

Com o objetivo de identificar a relação do domínio teórico e empírico do conteúdo dos itens da Escala de Atitude Valorativa Frente a Tecnologia (EAVTICs) verificou-se, a discriminação e representatividade do conteúdo dos itens. De acordo com Formiga, Fleury, Fandiño e Souza (2016), a função destas análises é de verificar, empírica e sistematicamente o quanto a proposta teórica elaborada para definição dos itens tem da escala e as situações especificadas nos itens e o quanto eles representam os aspectos esperados, bem como, garanta a relação comportamento-domínio do conteúdo dos itens na EAVTICs.

Para tal análise, orientou-se a partir dos pressupostos da Teoria Clássica dos Testes (TCT), os quais, têm como direção reflexiva, a avaliação dos itens quanto à sua capacidade em discriminar as respostas dos participantes da pesquisa relacionadas às proximidades das magnitudes dos grupos com pontuações inferiores e superiores com relação ao construto que se pretende mensurar; considerando com isso, que os possíveis erros de medidas, seriam independentes de qualquer circunstâncias, pois, o que se pretender avaliar, neste momento, é apenas o escore de uma medida ou teste, a qual, tem como referência a quantidade de itens respondidos, supostamente, corretos pelos participante do estudo. (Couto; Primi, 2011; Pasquali, 2011; Sartes; SouzaFormigoni, 2013).

Desta maneira, a fim de atender a essa condição hipotético-metodológica (a discriminação dos itens das escalas), gerou-se uma pontuação total para a medida em questão, calculou-se a sua mediana e organizou um grupo de respondentes que apresentaram pontuação abaixo da mediana (isto é, o grupo inferior) e outro grupo com pontuações acima foram definidos como do grupo superior (Formiga; Fleury; Fandiño; Souza, 2016).

Para cada um dos dez itens da escala realizou-se um teste t de Student para amostras independentes, passando a comparar os escore médios de ambos os grupos, os quais, foram capazes em discriminar cada um dos itens para as pessoas com magnitudes próximas, no grupo superior e inferior, estatisticamente significativas. É preciso destacar que, para a realização desses cálculos, uma amostra inicial com 50 participantes foi coletada, a qual, tinham semelhantes características sociodemográficas da amostra total destinadas ao estudo.

Na Tabela 1, pode-se observar o poder discriminativo dos itens na EAVTICs foram significativos, sendo assim, capazes de discriminar as pessoas com magnitudes próximas, estatisticamente significativas. Na mesma tabela, observou-se também, o IDi (Índice de Discriminação); associado aos cálculos do teste t de Student, tal indicador avalia a capacidade de diferenciar indivíduos com bom desempenho daqueles com baixa desempenho nas respostas aos itens da EAVTICs.

Neste índice, de acordo com Arias, Lloreda e Lloreda (2006), é preciso considerar os seguintes critérios classificatórios para análise da qualidade discriminativa dos itens, a saber: ID $\leq 0,20$ Ineficiente (Sugere-se eliminá-lo ou revisá-lo totalmente), $0,20 \leq \mathrm{ID} \leq 0,30$ (é preciso ser revisado), $0,30 \leq \mathrm{ID} \leq 0,40$ (Aceitável, não sendo necessária uma revisão), e por fim, ID > 0,40 (Satisfatório, devendo permanecer na escala). 
Tabela 1: Discriminação dos itens da EAVTICs.

\begin{tabular}{|c|c|c|c|c|c|c|}
\hline Itens & Grupos & Média & d.p. & $\mathrm{t}$ & $\mathrm{ID} i$ & $\begin{array}{c}\text { Correlação de } \\
\text { Pearson (r) } \\
\text { [EAVTICs] }^{\#}\end{array}$ \\
\hline \multirow{2}{*}{ EAVTICs1 } & GI & 4,06 & 0,90 & \multirow{2}{*}{$-7,82 *$} & \multirow{2}{*}{0,44} & \multirow{2}{*}{$0,63^{*}$} \\
\hline & GS & 4,76 & 0,46 & & & \\
\hline \multirow{2}{*}{ EAVTICs2 } & GI & 3,28 & 1,13 & \multirow{2}{*}{$-10,81^{*}$} & \multirow{2}{*}{0,34} & \multirow{2}{*}{$0,67 *$} \\
\hline & GS & 4,53 & 0,66 & & & \\
\hline \multirow{2}{*}{ EAVTICs3 } & GI & 3,81 & 1,01 & \multirow{2}{*}{$-9,91 *$} & \multirow{2}{*}{0,48} & \multirow{2}{*}{0,75} \\
\hline & GS & 4,77 & 0,42 & & & \\
\hline \multirow{2}{*}{ EAVTICs4 } & GI & 3,69 & 0,96 & \multirow{2}{*}{$-9,36^{*}$} & \multirow{2}{*}{0,39} & \multirow{2}{*}{$0,73 *$} \\
\hline & GS & 4,61 & 0,56 & & & \\
\hline \multirow{2}{*}{ EAVTICs5 } & GI & 3,55 & 1,00 & \multirow{2}{*}{$-9,37 *$} & \multirow{2}{*}{0,39} & \multirow{2}{*}{$0,78 *$} \\
\hline & GS & 4,68 & 0,51 & & & \\
\hline \multirow{2}{*}{ EAVTICs6 } & GI & 3,49 & 1,06 & \multirow{2}{*}{$-11,41^{*}$} & \multirow{2}{*}{0,40} & \multirow{2}{*}{$0,77 *$} \\
\hline & GS & 4,66 & 0,54 & & & \\
\hline \multirow{2}{*}{ EAVTICs7 } & GI & 3,34 & 0,98 & \multirow{2}{*}{$-11,08 *$} & \multirow{2}{*}{0,33} & \multirow{2}{*}{$0,78 *$} \\
\hline & GS & 4,60 & 0,55 & & & \\
\hline \multirow{2}{*}{ EAVTICs8 } & GI & 3,70 & 1,05 & \multirow{2}{*}{$-12,70^{*}$} & \multirow{2}{*}{0,35} & \multirow{2}{*}{$0,72 *$} \\
\hline & GS & 4,63 & 0,63 & & & \\
\hline \multirow{2}{*}{ EAVTICs9 } & GI & 3,71 & 1,15 & \multirow{2}{*}{$-8,66^{*}$} & \multirow{2}{*}{0,42} & $061 *$ \\
\hline & GS & 4,54 & 0,69 & & & \\
\hline EAVTICs1 & GI & 3,70 & 1,06 & & 044 & $073 *$ \\
\hline 0 & GS & 4,63 & 0,63 & $-7,02 *$ & 0,44 & $0,13^{*}$ \\
\hline
\end{tabular}

Nota: $* \mathrm{p}<0,01 ; \mathrm{r}=$ correlação de Pearson; $\mathrm{t}=$ teste de Student

ID $i=$ Índice de discriminação dos itens; \# Escala de Atitude

Valorativa Frente a Tecnologia (EAVTICs). Fonte: Autores.

Tendo a análise da discriminação dos itens sido significativa para todos eles, bem como, seus índices de discriminação se encontrado no parâmetro estabelecido, realizou-se o cálculo de representatividade de conteúdo itens-fator, a qual, é avaliada através do cálculo de correlação (r) de Pearson.

Na mesma tabela, é possível observar que tanto houve uma relação positiva dos itens da escala com a pontuação total da EAVTICs, quanto a estas, elas foram significativas e estiveram acima de 0,50. Destaca-se também, a existência de correlações intra-itens, as quais variaram de 0,37 a 0,62 .

Chama-se a atenção para a seguinte condição: esperava-se que as correlações estivesse acima de 0,50 quando se avaliasse itens-pontuação total (Formiga; Fleury; Fandiño; Souza, 2016).

O motivo em considerar o limite mínimo deste escore correlacional para a avaliação da representatividade de conteúdo, deve-se ao fato de encontrar na literatura sobre a fixação deste critério de intervalo de interpretação, a possibilidade de se afirmar uma aproximação empírica entre as variáveis, as quais, hipotetizadas teoricamente, garantem a qualidade de que o conteúdo do item converge para a condição expressa no item com base na definição do construto (Pasquali, 2011; Figueiredo Filho; Silva Junior, 2010; Cohen; Swerdlik; Sturman, 2014; Soares; Silva; Chariglione; Formiga; Melo, 2019).

Chama-se atenção para o IDi na discriminação dos itens, os quais, estiveram no padrão aceitável, proposto por Arias, Lloreda e Lloreda (2006), em relação qualidade dos itens na escala EAVTICs. Considerados os resultados destacados acima, observados nas duas análises anteriores, realizou-se uma análise fatorial exploratória (AFE). Nesta etapa, participaram do estudo 228 respondentes, todos estudantes universitários na cidade de Natal-RN, distribuídos

Para a realização da AFE, estabeleceram-se os seguintes critérios: o método dos Componentes Principais (PC), com a proposta de número de fatores a se extrair sem fixação da quantidade destes, escolha da rotação fatorial oblíqua (oblimin) para a fatorialização e o pontuação mínima da saturação fatorial estabelecida como sendo $\geq 0,30$ para a retenção dos fatores 
(Dancey; Reidy, 2006).

Visando uma maior qualidade e segurança para uma tomada de decisão na escolha dos fatores, procurou-se atender aos critérios para a avaliação fatorial: (1) quantidade de valores próprios (eigenvalues) $\geq 1,00$ (Critério de Kaiser), (2) distribuição gráfica dos valores próprios, isto é, o gráfico de declividade, no qual, pode-se indicar, quanto referência, o ponto do corte a partir que limita a quantidade de fatores consideráveis para a estrutura fatorial (Critério de Cattell) e (3) análise paralela (Formiga; Freire; Fernandes, 2019).

Com base nestes critérios, os resultados da AFE permitiram identificar a adequação da matriz de correlação: $\mathrm{KMO}=$ 0,91 e do Teste de Esfericidade de Bartlett, $\chi^{2} / \mathrm{gl}=1230,02 / 45, p<0,001$. Na distribuição dos valores próprios (critério de Cattell), dois fatores foram identificados com os valores próprios maiores do que 1,00 (ver Figura 1). É possível observar na figura, o ponto de corte desse critério, confirmando a existência da conglomeração desses dois fatores.

Figura 1: Diagrama de declividade do número de fatores plotados da EAVTICs.

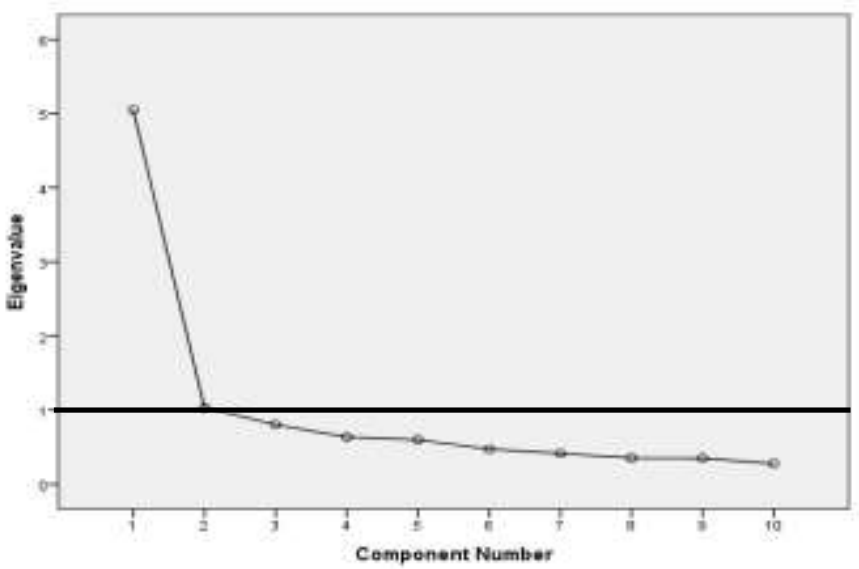

Fonte: Autores.

No resultado do critério de Kaiser (Eigenvalues), foi também, observada a existência de dois fatores com valores próprios maiores que 1 (um), explicando, conjuntamente, $60,82 \%$ da variância total da referida medida. O indicador do Eigenvalues está fixado na fatorialização, especificamente, em 1,03, os qual é bem menor do que o observado no primeiro fator, que foi de 5,04; para não deixar dúvidas referente à organização fatorial, realizou-se o cálculo da análise paralela, assumindo assim, o mesmo parâmetro do banco de dados original (208 participantes e 10 variáveis-itens relativo a escala EAVTICs) e com seus valores próprios gerados em 1.000 simulações aleatórias com os itens (Ver Tabela 2).

Tabela 2: Distribuição dos fatores da análise PC de acordo com o critério de Kaiser e análise paralela.

\begin{tabular}{c|c}
\hline Critério de Kaiser & Análise paralela \\
\hline $\mathbf{5 , 0 4}$ & $\mathbf{1 , 4 4}$ \\
\hline 1,03 & 1,31 \\
\hline
\end{tabular}

Fonte: Autores.

Ao comparar os escores dos valores próprios, observados nos critérios de Kaiser e os da análise paralela, pode-se afirmar que no resultado encontrado no critério de Kaiser, apenas um fator apresentou valor superior ao simulado (isto é, o da análise paralela). Para a fatorialização da EAVTICs, a organização uni-fatorial é a mais recomendada para a realização da AFE. 
Ainda assim, para oferecer maior garantia para a distribuição item-fator, juízes especializados (no conhecimento da estatística, construção e avaliação de instrumentos) foram consultados, para avaliar a decisão tomada na pesquisa.

Atento a interpretação do item-fator estabelecida pelos juízes, confirmando os achados na comparação do resultado no critério de Kaiser e da análise paralela, estes, contribuíram à proposta uni-fatorialidade da escala.

Desta forma, procedeu-se, de forma mais segura, a realização da análise fatorial dos Componentes Principais (PC) com rotação oblíqua, eigenvalue (valor próprio) $\geq 1,00$ para definir o fator e saturação de $\geq 0,30$ para retenção dos itens.

A utilização desta técnica se mostrou bastante adequada, tendo os indicadores estatísticos (KMO = 0,91 e do Teste de Esfericidade de Bartlett, $\left.\chi^{2} / \mathrm{gl}=1230,02 / 45, p<0,001\right)$ revelado a possibilidade de que a fatorialização fosse realizada sem nenhum problema para a análise estatística.

Os resultados da extração fatorial, revelaram que a EAVTICs tem sua organização em um único fator, seguindo a direção do resultado encontrado nas análises estatísticas iniciais, para decisão fatorial, da tomada de decisão - valores próprios (eigenvalues) iguais ou superiores a 1 (Critério de Kaiser), distribuição gráfica dos valores próprios (Critério de Cattell) e a análise paralela - revelando uma organização lógica relativa a associação item-fator e com os escores fatoriais estiveram acima do padrão exigido $(\geq 0,50)$ (ver Tabela 3).

Tabela 3: Análise fatorial dos principais componentes EAVTICs.

\begin{tabular}{|c|c|c|c|c|}
\hline Itens & $\begin{array}{l}\text { Média } \\
\text { (d.p.) }\end{array}$ & SK & EAVTICs & $\mathbf{h}^{2}$ \\
\hline EAVTICs 1 & $\begin{array}{c}4,47 \\
(0,82)\end{array}$ & $-1,54$ & 0,80 & 0,44 \\
\hline EAVTICs 2 & $\begin{array}{c}3,83 \\
(1,14) \\
\end{array}$ & $-0,88$ & 0,77 & 0,42 \\
\hline EAVTICs 3 & $\begin{array}{c}4,23 \\
(0,94) \\
\end{array}$ & $-1,46$ & 0,77 & 0,60 \\
\hline EAVTICs 4 & $\begin{array}{c}4,10 \\
(0,93) \\
\end{array}$ & $-1,11$ & 0,76 & 0,55 \\
\hline EAVTICs 5 & $\begin{array}{c}4,05 \\
(0,99)\end{array}$ & $-1,09$ & 0,75 & 0,63 \\
\hline EAVTICs 6 & $\begin{array}{c}4,01 \\
(1,05)\end{array}$ & $-0,96$ & 0,74 & 0,57 \\
\hline EAVTICs 7 & $\begin{array}{c}3,90 \\
(1,03) \\
\end{array}$ & $-0,86$ & 0,70 & 0,59 \\
\hline EAVTICs 8 & $\begin{array}{c}4,11 \\
(1,01) \\
\end{array}$ & $-1,16$ & 0,66 & 0,56 \\
\hline EAVTICs 9 & $\begin{array}{c}4,08 \\
(1,06) \\
\end{array}$ & $-1,12$ & 0,65 & 0,50 \\
\hline EAVTICs 10 & $\begin{array}{c}4,11 \\
(1,01)\end{array}$ & $-1,16$ & 0,54 & 0,61 \\
\hline Número total de itens & \multicolumn{4}{|l|}{10} \\
\hline Valores próprios & \multicolumn{4}{|l|}{5,05} \\
\hline Variância explicada & \multicolumn{4}{|l|}{$50,49 \%$} \\
\hline Alfa de Cronbach & \multicolumn{4}{|l|}{$0,88 *$} \\
\hline $\begin{array}{l}\text { Alfa de Cronbach (com } \\
\text { exclusão dos itens) }\end{array}$ & \multicolumn{4}{|c|}{$0,86-0,87$} \\
\hline $\begin{array}{l}\text { ICC } \\
(95 \% \text { IC })\end{array}$ & \multicolumn{4}{|c|}{$\begin{array}{l}0,88^{*} \\
(0,86-0,90)\end{array}$} \\
\hline $\mathrm{F}$ (teste Friedman) & \multicolumn{4}{|l|}{$63,55^{*}$} \\
\hline Escore médio do construto & \multicolumn{4}{|c|}{$\begin{array}{l}39,67 \\
(\text { d.p. }=7,18)\end{array}$} \\
\hline
\end{tabular}

Nota: ${ }^{*} \mathrm{p} \geq 0,001 ;$ EAVTICs $=$ Escala de Atitude Valorativa Frente a Tecnologia da informação. Fonte: Autores. 
Na Tabela 1, é possível avaliar a distribuição unifatorial dos itens da EAVTICs; isto é, um único fator foi capaz de mensurar a atitude de valoração frente a Tecnologias da Informação e Comunicação, a qual, poderá ser definida como, comportamento e pensamento dos universitários a respeito do valor dado pelos próprios quanto ao uso de tecnologia da informação para a motivação e engajamento da prática discente no ambiente de sala de aula nas IES.

A mesma tabela, apresentou a distribuição dos itens por fator, sua saturação (carga fatorial) e comunalidade, bem como, os indicadores de consistência interna (Alfa de Cronbach), variância explicada pelo fator e a correlação intra-classe (ICC). Todos os indicadores estiveram dentro do padrão psicométrico exigido (Hair; Tatham; Anderson; Black, 2005; Pasquali, 2011), o que garante a qualidade fatorial da escala e sua confiança frente ao que se pretendeu mensurar em um grupo de universitários.

Pode-se também, observar que os escores médios dos itens, na análise fatorial, tenderam para o extremo concordante da escala de medida, bem como, o indicador de assimetria (Skewness - Sk), se encontrar no intervalo estatístico exigido, isto é, abaixo de 2,00.

Raciocínio semelhante poderá ser observado, no escore médio do fator, o qual, apresentou uma média de 35,60 (d.p.= 6,47), distribuída na variação dos escores de 9 a 45 pontos. Esta distribuição média se encontra dentro da normalidade, pois, apresentou uma Assimetria (Skewness - Sk) e Curtose (Kurtosis - Ku) (respectivamente, -1,23 e 1,23), correspondente ao parâmetro considerado na literatura estatística quanto aceitável.

Com base nos resultados apresentados acima, optou-se em avaliar as frequências das respostas entre os respondentes em função das variáveis propostas neste artigo. Desta maneira, realizou-se o cálculo do qui-quadrado, buscando verificar a associação entre idade, origem universitária, tipo de curso, período do curso em função da EAVTICs.

Para efetuar o cálculo do qui-quadrado, com objetivo de responder às comparações das frequências entre as variáveis, inicialmente, realizou-se uma análise descritiva, tendo em conta a análise de tercis, através da qual, se dividiram as variáveis em níveis categoriais, o construto EAVTICs, quanto variável contínua passou a ser categorizada em três níveis: baixa, moderada e alta atitude valorativa das TIC's. Em seguida, procedeu-se à análise da distribuição da amostra, considerando as variáveis em estudo.

$\mathrm{Na}$ Tabela 4, pode-se observar uma maior frequência para os respondentes que estão acima de 60 anos, porém, além de ser um resultado ambivalente, pois, $50 \%$ da frequência de respostas foi observada tanto para baixa quanto alta atitudes valorativa para esse grupo etário, bem como, é preciso considerar que esses resultados não foram significativos.

Tabela 4: Frequência em percentagem da faixa etária em função da EAVTICs em universitários.

\begin{tabular}{|c|c|c|c|c|c|c|}
\hline \multirow{2}{*}{ Idade } & \multicolumn{3}{|c|}{ Nível Atitude valorativa das TIC's } & \multicolumn{3}{|c|}{ Estatística } \\
\hline & Baixo & Moderado & Alto & $\chi^{2}$ & $\mathrm{gl}$ & $\begin{array}{l}\text { p- } \\
\text { valor }\end{array}$ \\
\hline 17 a 21 anos & $42 \%(18)$ & $37 \%(16)$ & $21 \%(9)$ & \multirow{5}{*}{4,90} & \multirow{5}{*}{8} & \multirow{5}{*}{0,77} \\
\hline 22 a 29 anos & $38 \%(38)$ & $31 \%(31)$ & $31 \%(31)$ & & & \\
\hline 30 a 39 anos & $32 \%(30)$ & $34 \%(32)$ & $35 \%(33)$ & & & \\
\hline 40 a 59 anos & $32 \%(12)$ & $40 \%(15)$ & $29 \%(11)$ & & & \\
\hline Acima de 60 anos & $50 \%(1)$ & $0 \%(0)$ & $50 \%(1)$ & & & \\
\hline
\end{tabular}

Fonte: Autores.

$\mathrm{Na}$ Tabela 5, é apresentado o resultado da frequência dos respondentes associando curso e o nível de atitude valorativa dos TIC's. Considerando que os resultados são significativos, observou-se que curso de Engenharia (67\%) dos alunos que cursam tem uma baixa atitude às TIC's. 
Tabela 5: Frequência em percentagem do curso que fazem em função da EAVTICs em universitários.

\begin{tabular}{|c|c|c|c|c|c|c|}
\hline \multirow[t]{2}{*}{ Curso } & \multicolumn{3}{|c|}{$\begin{array}{l}\text { Nível Atitude valorativa das } \\
\text { TIC's }\end{array}$} & \multicolumn{3}{|c|}{ Estatística } \\
\hline & Baixo & Moderado & Alto & $\chi^{2}$ & $\mathrm{gl}$ & $\begin{array}{l}\mathrm{p}- \\
\text { valor }\end{array}$ \\
\hline Administração & $\begin{array}{l}26 \% \\
(15)\end{array}$ & $\begin{array}{l}48 \% \\
(16)\end{array}$ & $\begin{array}{l}26 \% \\
(15)\end{array}$ & \multirow{5}{*}{16,53} & \multirow{5}{*}{8} & \multirow{5}{*}{0,05} \\
\hline $\begin{array}{l}\text { Ciências } \\
\text { Contábeis }\end{array}$ & $\begin{array}{l}47 \% \\
(51) \\
\end{array}$ & $\begin{array}{l}28 \% \\
(31)\end{array}$ & $\begin{array}{l}33 \% \\
(44) \\
\end{array}$ & & & \\
\hline Engenharia & $\begin{array}{l}67 \% \\
(12) \\
\end{array}$ & $\begin{array}{l}17 \% \\
(32) \\
\end{array}$ & $\begin{array}{l}37 \% \\
(3)\end{array}$ & & & \\
\hline Tecnólogo & $\begin{array}{l}27 \% \\
(4)\end{array}$ & $\begin{array}{l}33 \% \\
(5)\end{array}$ & $\begin{array}{l}40 \% \\
(6)\end{array}$ & & & \\
\hline Outros & $\begin{array}{l}32 \% \\
(17)\end{array}$ & $\begin{array}{l}39 \% \\
(21)\end{array}$ & $\begin{array}{l}19 \% \\
(16)\end{array}$ & & & \\
\hline
\end{tabular}

Fonte: Autores.

Seguindo a ordem das variáveis, na tabela 6 , pode ser observada a frequência em percentagem das respostas dos participantes relacionando período do curso e o nível de atitude valorativa dos TIC's. Considerando que os resultados são significativos, observou-se que existe um aumento no percentual entre o período do curso, assim, do meio para o final do curso a atitude valorativa apresenta um aumento baixo de adesão às TIC's.

Tabela 6: Frequência em percentagem do período do curso em função da EAVTICs em universitários.

\begin{tabular}{|c|c|c|c|c|c|c|}
\hline \multirow{2}{*}{ Período } & \multicolumn{3}{|c|}{ Nível Atitude valorativa das TIC's } & \multicolumn{3}{|c|}{ Estatística } \\
\hline & Baixo & Moderado & Alto & $\chi^{2}$ & gl & $\begin{array}{l}\mathrm{p}- \\
\text { valor }\end{array}$ \\
\hline Início & $30 \%(57)$ & $\begin{array}{l}37 \% \\
(71)\end{array}$ & $33 \%(62)$ & \multirow[t]{3}{*}{10,95} & \multirow[t]{3}{*}{4} & \multirow[t]{3}{*}{0,01} \\
\hline Meio & $47 \%(21)$ & $\begin{array}{l}33 \% \\
(15) \\
\end{array}$ & $20 \%$ (9) & & & \\
\hline Fim & $49 \%(21)$ & $\begin{array}{l}19 \% \\
(8)\end{array}$ & $33 \%(14)$ & & & \\
\hline
\end{tabular}

Fonte: Autores.

Com base nos resultados da Tabela 6, procurou-se avaliar as associações por especificidade dos períodos e a atitude valorativa dos TIC's. Desta maneira, na Tabela 7, pode-se observar que no primeiro e segundo períodos há uma valoração nos níveis de adesão as TIC's; porém, esse resultado decresce quando os períodos avançam com o tempo no decorrer do curso. Vale destacar que esses resultados foram significativos.

Tabela 7: Frequência em percentagem da especificidade do período do curso em função da EAVTICs em universitários.

\begin{tabular}{|c|c|c|c|c|c|c|}
\hline \multirow{2}{*}{$\begin{array}{l}\text { Especificidades } \\
\text { dos Períodos de } \\
\text { curso }\end{array}$} & \multicolumn{3}{|c|}{ Nível Atitude valorativa das TIC's } & \multicolumn{3}{|c|}{ Estatística } \\
\hline & Baixo & Moderado & Alto & $\chi^{2}$ & $\mathrm{gl}$ & $\mathrm{p}$ \\
\hline Primeiro & $32 \%(34)$ & $40 \%(43)$ & $29 \%(31)$ & \multirow{6}{*}{17,9} & \multirow{6}{*}{10} & \multirow{6}{*}{0,05} \\
\hline Segundo & $28 \%(23)$ & $34 \%(28)$ & $38 \%(31)$ & & & \\
\hline Terceiro & $52 \%(13)$ & $40 \%(10)$ & $8 \%(2)$ & & & \\
\hline Quarto & $40 \%(8)$ & $25 \%(5)$ & $35 \%(7)$ & & & \\
\hline Quinto & $55 \%(11)$ & $10 \%(2)$ & $35 \%(7)$ & & & \\
\hline Outros & $44 \%(10)$ & $26 \%(6)$ & $30 \%(7)$ & & & \\
\hline
\end{tabular}

Fonte: Autores. 
Por fim, na Tabela 8, pode ser observada a frequência em percentagem, relacionando IES e nível de atitude valorativa dos TIC's. Apesar de existir um maior percentual na baixa adesão as TIC's na universidade pública, esses resultados não são significativos. Mesmo com pouca confiança, merece ser destacado que há uma distribuição, equitativa, nos níveis da atitude valorativa das TIC's na IES privada.

Tabela 8: Frequência em percentagem do período do curso em função da EAVTICs em universitários.

\begin{tabular}{|c|c|c|c|c|c|c|}
\hline \multirow{2}{*}{ IES } & \multicolumn{3}{|c|}{ Nível atitude valorativa das TIC's } & \multicolumn{3}{|c|}{ Estatística } \\
\hline & Baixo & Moderado & Alto & $\chi^{2}$ & $\mathrm{gl}$ & $\begin{array}{l}\mathrm{p}- \\
\text { valor }\end{array}$ \\
\hline Privada & $33 \%(73)$ & $36 \%(78)$ & $31 \%(69)$ & \multirow[b]{2}{*}{3,24} & \multirow[b]{2}{*}{2} & \multirow[b]{2}{*}{0,19} \\
\hline Pública & $46 \%(26)$ & $26 \%(15)$ & $28 \%(16)$ & & & \\
\hline
\end{tabular}

Fonte: Autores.

\section{Resultados e Discussão}

Neste artigo avaliou-se uma escala destinada a mensuração de atitudes valorativas dos discentes frente às TICs. A intenção em desenvolver um estudo nesta direção, se deve ao fato de não existir um instrumento nesta direção avaliativa para o contexto do ensino superior, bem como, por ser a TICs um elemento pedagógico e didático, a ser utilizado, nos últimos anos, com bastante frequência destinado ao processo ensino-aprendizagem de forma mais abrangente.

Considerando os resultados referentes à escala de medida proposta para mensurar a valoração frente a tecnologia da informação e comunicação aplicada na sala de aula, pode-se afirmar que a análise de conteúdo dos itens, seja em relação a discriminação e a sua representatividade, atenderam ao critério de análise estatística relativo ao conteúdo e domínio teórico da medida proposta. Estes, estiveram de acordo com a proposta empírica estabelecida por Pasquali (2011), o qual, afirma que medidas 'originais' deverão apresentar condição psicométrica que permitam afirmar a existência de uma qualidade inicial do instrumento ministrado para os respondentes, sejam aqueles que discordam, sejam os que concordam, de forma que, representativamente, indique que os participantes reconheceram o conteúdo dos itens quando o instrumento foi administrado a eles.

Sendo assim, o autor supracitado, sugere que, para a discriminação, com a análise do teste t de Student, seja significativo e com um $\mathrm{t}>1,96$, e para a representatividade de conteúdo, a partir da observação da correlação de Pearson (r), a qual, apresente um ' $r$ ' $>0,50$ e p < 0,001. Condições, as quais, permitem afirmar que neste estudo, os itens e sua relação com o construto, em todas as questões apresentadas, tornaram-se importantes para a formação do instrumento.

$\mathrm{Na}$ análise fatorial, a escala proposta sobre atitude valorativa frente a tecnologia, apresentou garantias para a sua distribuição unifatorial, revelando que não apenas esse fator faz parte de uma estrutura fatorial confiável, mas, que foi fidedigna, ao avaliar o alfa e o ICC, podendo ser administrada numa amostra de universitários, de IES públicas e privadas, para avaliação das TICs.

Ao considerar estes resultados, é preciso destacar que não se trata apenas de um instrumento atitudinal e ou de avaliação educacional em IES, simplesmente, para verificar a qualidade de um instrumento de medida; acredita-se que se trata de uma perspectiva diagnóstica de uma pedagogia universitária bem mais ampla. O que é proposto com esta medida, é uma avaliação mais segura do sentido e significado das TICs no contexto universitário, já que, parece ser, uma prática irreversível para os novos contextos sociais, econômicos e acadêmicos (Damm; Tonetto; Riedner; Pischetola, 2016).

Um fator primordial para a proposta desta medida, afim de torná-la mais um instrumento no contexto educativo na avaliação dos fenômenos da educação superior, é tratá-lo quanto orientação e indicadores aplicáveis aos ajustes de práticas e ideais para uma educação superior mais ágil, motivadora e contemporânea; bem como, para a orientação do desenvolvimento 
de uma didática destinada à identificação da autoeficácia na relação professor-aluno-IES, no uso dessas tecnologias e seu possível enriquecimento intelectual para formação universitária (Jenkins; Clinton; Purushotma; Robison; Weige, 2009; Pischetola, 2016).

Em relação à escala de medida em questão, se apresentar numa organização unifatorial, esta, corrobora com os estudos de Chen, (2010) e Chien et al., (2012). De acordo com estes autores, o uso da tecnologia pelos professores facilita a inovação e a motivação, as quais, não poderão ser mensuradas de forma separadas, mas, acredita-se que estão bastante relacionadas no espaço etno-aula (Camacho, 2017; Wiebusch; Lima, 2018).

Tal condição, apresentada na escala, sugere nas questões propostas no instrumento, uma direção didático-pedagógica, para que permita ao docente ou discente, estarem preparados para usar efetivamente a tecnologia em suas práticas diárias de sala de aula, mais, como um aliado do que quanto concorrência negativa.

Quanto elemento avaliador, a escala, é capaz de gerar nos discentes, não apenas uma mudança na percepção dos novos processos motivadores e inovadores em sala de aula, quando o docente utiliza das TICs para o aperfeiçoamento e a compreensão do aprendizado de sala de aula, mas, também, para conduzir para uma proposta de formação educacional mais flexível, quanto ao acesso desta aos discentes que buscam ingressar de forma remota, nas atividades de cultural e formação acadêmica com qualidade.

\section{Considerações Finais}

Em termos gerais, as intenções em um processo mais tecno-contemporâneo da educação superior não poderão ser avaliadas de forma separada, item a item, mas, no conjunto de um construto, o qual, contempla um conglomerado de possibilidades (expressos nos itens da escala) práticas, variando apenas em intensidade e frequência, mas, que poderão ser encontradas, provavelmente, quando o professor for motivado a empregar as TICs (cf. Pasquali, 2011).

Sendo assim, a escala relacionada à atitude valorativa frente à tecnologia (TICs ao ser utilizada pelo professor, na concepção dos respondentes) é capaz, em sala de aula, de explorar diferentes perspectivas, para as quais, destinadas as maneiras de analisar os problemas de forma mais rápida e entusiasmada para compreensão dos alunos e, consecutivamente, maior desenvolvimento cultural, intelectual e acadêmico.

Tais resultados, corroboram aos desenvolvidos por Enochsson e Rizza (2009), Kumar e Vigília (2011) e Stobaugh e Tassel (2011), os quais, indicam que a assistência no uso da tecnologia pelos professores na sala de aula, associando a confiança na capacidade destes a usarem. Considerando os resultados nas análises de frequência, alguns resultados chamam a atenção; especificamente, quando se avaliou a idade e o tipo de IES, pois, os resultados não foram significativos.

Afinal, acreditava-se que os discentes mais novos apresentassem maiores percentuais de adesão as TICs, mas, por outro lado, observou-se que os respondentes acima de 40 anos, revelaram ser mais aderentes, revelando escores mais alto na moderada e alta adesão quando comparado aos mais jovens (ver tabela 1). Este resultado representa algo bastante curioso para estes percentuais, pois, as TICs têm uma dinâmica e aplicação na tradicional, exigindo conhecimentos em áreas da tecnologia e lógica relacionado a dimensão cognitiva e motriz do respondente.

Uma outra situação, relacionada a não existência de diferenças da adesão ás TICs nas IES; esperava-se que as IES privadas apresentassem maior frequência de adesão, o que não aconteceu. Esse fato poderá ser compreendido em relação aos critérios de implementação, treinamento e utilização dos mecanismos tecnológicos com maior facilidade, seja por parte do discente, docente e até das IES, pois, o uso dos instrumentos tecnológicos (celular, computador, tablete, etc.) é destinado mais para o uso pessoal de lazer e comunicação na troca de mensagens do que, de fato, aplicada ao processo de formação acadêmica contínua. 
Um dos resultados significativos que chamou a atenção foi em relação aos períodos de curso dos respondentes. Enquanto os discentes do primeiro período, apresentaram uma moderada adesão, o maior percentual foi observado para aqueles dos últimos períodos em relação a baixa adesão.

Com isso, ao considerar os resultados apresentados na tabela 6, poderá ser compreendido a partir dos seguintes pontos: por ser uma ferramenta pedagógica ainda muito recente, é possível que as instituições ainda não socializaram e implementaram $100 \%$ esta prática na dinâmica acadêmica delas, pois, caso contrário, o resultado seria bastante positivo, já que a habilidade nesta ferramentas dependem da implementação, divulgação, treinamento e uso pessoal diário; uma outra explicação, estaria associada a dimensão mais prática, exigida no final do período que os respondentes experimentam no final do curso, o que geralmente, é atribuído a atividade formativa de teor mais prático.

A explicação destacada acima, fica mais clara quando se observa, na tabela 5, a comparação da adesão a TICs e o curso que os respondentes fazem. É destacável o maior percentual na baixa adesão pelos discentes do curso de engenharia; este, por sua vez, trata-se de uma formação com, praticamente, $70 \%$ de atividades práticas e de campo. Associado a tal resultado, poderia complementar-se este raciocínio, considerando o percentual observado na tabela 6 , pois, os discentes dos últimos períodos (meio e fim) foram os que tiveram maiores percentuais na baixa adesão.

Com isso, estes resultados refletem-se na seguinte direção: no que diz respeito à qualidade da escala, esta, poderia ser considerada como um elemento de medida e diagnose importante para as IES referente a adesão às TICs; uma outra questão, é quanto à importância das IES investirem mais em um treinamento e socialização das TICs na internalidade dos espaços pedagógicos delas, formando e treinando docentes e discentes. Por fim, uma terceira reflexão, trata do desenvolvimento de políticas e práticas institucionais mais efetivas, destinadas aos últimos períodos, pois, já existem programas computacionais e virtuais que facilitam o processo de aprendizagem para os cursos mais técnicos e aqueles que utilizam de alguns laboratórios para melhor aprendizagem e assimilação do conteúdo destinado a formação profissional competente e eficaz.

A adoção das TIC por estudantes universitários é favorecida cada vez mais por uma série de fatores da própria evolução tecnológica existente, haja vista que veem as TICs não somente como uma tecnologia isolada, mas como o processo de ensino- aprendizagem dentro da sala de aula.

Isso ocorre porque, em muitas instituições de ensino superior, o uso de TIC, como mecanismos de busca na Internet, substituiu não somente a biblioteca, mas também vem substituindo a própria forma de aprendizado e a forma do docente ministrar aulas. A inserção das TICs no cotidiano universitário, estimula o desenvolvimento do pensamento crítico, criativo e da aprendizagem cooperativa, uma vez que torna possível a realização de atividades interativas conforme observado no resultado das tabelas 7 e 8 .

Desta forma observou-se na pesquisa que as tecnologias de informação e comunicação funcionam como recursos dinâmicos de aprendizado colaborativo a partir da percepção dos alunos conforme demonstrado nas tabelas 6,7 e 8 , invariavelmente pelo curso, idade ou período matriculado. Sendo assim, esta escala pode ser facilmente aplicada em outro grupo de pessoas, outras cidades ou localidade, já que a medida conforme Tabelas 2, 3 e 4 não houve incoerências estatística que inviabilizasse o mesmo.

É preciso compreender que a ferramenta tecnológica não é ponto fundamental no processo de ensino e aprendizagem, mas, um dispositivo que proporciona a mediação entre educador e educando, diante disso, temos que entender que, a inserção das TICS no ambiente educacional, depende primeiramente da formação do professor em uma perspectiva que procure desenvolver uma proposta que permita transformar o processo de ensino em algo dinâmico e desafiador com o suporte das tecnologias.

Desta maneira, as TICs quando articuladas a uma prática formativa que leva em conta os saberes trazidos pelo aluno, associando os conhecimentos, se tornam essenciais para a construção dos saberes, fruto para o resultado do último objetivo 
específico que trouxe nos resultados apresentados a aferição, a partir da análise fatorial exploratória, a organização dos fatores e seu grau de consistência interna para validar os resultados apresentados da relação das TICs com o ensino colaborativo na percepção dos alunos.

Sugere-se uma alternativa para estudos futuros: melhorias ou evolução do modelo apresentado a fim de se trazer perspectivas pedagógicas para os diversos índices de aprendizagem com o uso de TICs.

Este estudo também recomenda considerar a percepção dos professores das universidades, haja visto que para os professores que contribuem para a comodidade acadêmica dos discentes, uma investigação no futuro envolvendo estes atores podem trazer a literatura científica qual o papel crítico no auxílio ao processo de preparação das TICs para sala de aula.

Acredita-se, com base nos achados desta dissertação, que é necessário envolver todos o corpo de atores da IES envolvido neste processo educacional, sejam diretos ou indiretos ao processo. Portanto, este estudo recomenda que todas as partes se envolvam na sequência do estudo e compreendam os componentes das TIC. O estudo atual foi limitado em tempo, ambiente geográfico e físico; portanto, não foi possível estudar outras variáveis, como o uso das TIC.

Por fim, no que se refere a estudos futuros, seriam importante comparar instituições públicas e privadas em distintos estados brasileiros, bem como, identificar quais tem alta especialização nas TICs, buscando verificar a variação da aderências dos respondentes na escala; outra condição bastante útil seria o desenvolvimento de um estudo transcultural, com foco nas avaliação das similaridades e diferenças relacionada a sistemas de política educacional e universitária no uso das TICs. Outro estudo que poderia acrescentar informação nos resultados observados na dissertação seria a comparação das pontuações médias para uma amostra de professores e alunos.

\section{Referências}

Almekhlafi, A., Ismail, S., \& Al-Mekhlafy, M. H. (2017). Male and female language teachers technology integration differences in elementary schools in the United Arab Emirates. International Journal of Research Studies in Educational Technology. 6(1), 1-14. <https://doi.org/10.5861/ijrset>.

Almerich, G., Orellana, N., Suárez-Rodríguez, J., \& Díaz-García, I. (2016). Teachers information and communication technology competences: A structural approach. International Journal Computers e Education, 100, 110-125. <https://doi.org/10.1016/j.compedu.2016.05.002> .

Arias, M. R. M.; Lloreda, M. V. H.; \& Lloreda, M. J. H. (2006). Psicometría. Alianza Editorial, 488 p.

Barrette, C. (2017). Réussir l'intégration pédagogique des TIC, Bulletin Clic, Montréal, n 63.<http://clic.ntic.org/cgi-bin/aff.pl?page=article\&id=2020.

Blau, I., Shamir-Inbal, T. (2017). Digital competences and long-term ICT integration in school culture: The perspective of elementary school leaders. Education and Information Technologies, 22(3), 769-787. https://doi.org/10.1007/s10639-015-9456-7.

Bryman, A. (2012). Social Research Methods. (4th ed.), Oxford University Press.

Camacho, C. M. L. P. (2017). Recursos tecnológicos e motivação para a aprendizagem. Tese (Mestrado). Universidade Fernando Pessoa, Faculdade de ciências humanas e sociais.

Ciolan, L., Petrescu, A., Radulescu, C., \& Bucur, C. (2014). Training teachers to use digital resources for the knowledge society. Procedia-Social and Behavioral Sciences. 128, 415-419. <https://doi.org/10.1016/j.sbspro.2014.03.180>.

Corrêa, J. (2005) Sociedade da informação, globaliza Sociedade da informação, globalização e educação ação e educação a distância. Senac, p. 6.

Costa, C. S.; Mattos, F. R. P. (2016). Tecnologia na sala de aula em relatos de professores. Curitiba: CRV. 202 p. (Série: Recursos Didáticos Multidisciplinares, v. 1).

Creswell, J. W. (2013). Research design: qualitative, quantitative, and mixed methods approaches (4th ed.), Sage Publications.

Damm Tonetto Riedner, D., \& Pischetola, M. (2016). Tecnologias Digitais no Ensino Superior: uma possibilidade de inovação das práticas? Educação, Formação \& Tecnologias. <https://eft.educom.pt/index.php/eft/article/view/526>.

Dancey, C. P. \& Reidy, J. (2006). Estatística sem Matemática para Psicologia: Usando SPSS para Windows. Editora Artmed,

Demir, O., Genc, E. G., Alp, E. A., \& Yildirim, F. (2015). A New Knowledge Society Index: Global Tendencies and an Analysis of Turkey. Educational Sciences: Theory and Practice, 15(2), 325-335. <https://doi.org/10.12738/estp.2015.2.2356 .

Duart, J. M., \& Mengual-Andrés, S. (2014). Impact Of The Knowledge Society In The University And In Scientific Communication. RELIEVE-Revista Electrónica de Investigación y Evaluación Educativa. 20(2). <https://doi.org/10.7203/relieve.20.2.4343 
Enochsson, A.-B., \& Rizza, C. (2016). ICT in initial teacher training: Research review. OECD Publishing. <https://www.oecd.org/sweden/45046846.pdf>.

Esteban, M. P. S. (2010). Pesquisa qualitativa em educação: fundamentos e tradições. McGraw-Hill.

Fichten, C., Jorgensen, M., Havel, A., King, L., Lussier, A., Asuncion, J., \& Amsel, R. (2018). Information and Communication Technologies: Views of Canadian College Students and "Excellent" Professors. Journal of Education and Training Studies, 6(9), 1-12. <https://doi.org/10.11114/jets.v6i9.3390 .

Figueiredo, P. N. (2009) Capacidade Tecnológica e Inovação em Organizações de Serviços Intensivos em Conhecimento: evidências de institutos de pesquisa em Tecnologias da Informação e da Comunicação (TICs) no Brasil. Revista Brasileira de Inovação, 5(2), $403-454$.

Formiga, N. S., Fleury, L. F. O., Fandiño, A. M., \& Souza, M. A. (2016). Evidência empírica de uma medida da anomia organizacional em trabalhadores brasileiros. Revista de Psicologia da UCV. 18(1), 43-59. 10.18050/revpsi.v18n1a4

Fonseca, D. O. C. L. (2008). A formação de professores e a utilização das tecnologias de informação e comunicação nos cursos de licenciatura da Universidade do Estado da Bahia, Campus II. Tese (Doutorado). Universidade Federal da Bahia. Faculdade de Educação.

Francisco, C. (2011). Formação docente: O uso de conteúdos midiáticos e das TIC no processo de ensino e de aprendizagem no ensino superior. Acta Scientiarum. Education Maringá, 33, 9-55.

González Cubillán, Lesbia. (2009). Estudio de casos bajo el enfoque transdisciplinar. Multiciencias, Zulia, 9(3), 303-312.

Hair, J. F. Black, W. C. e Anderson, Rolph, \& Tatham, R. L. (2015). Análise multivariada de dados. https://www.researchgate.net/publica tion/304373193_Analise_multivariada_de_dados.

Hu, T. S. (2014) The implication of micro-teaching and its teaching design. Journal of Guangdong Education. <https://www.semanticschola r.org/paper/f9ed1698126ab2a1976d3d0f01f50890f9e7cf22?p2df>.

Jenkins, H.; Clinton, K.; Purushotma, R.; Robison, A. J.; \& Weigel, M. (2009) Confronting the Challenges of Participatory Culture: Media Education for the 21 st Century. Cambridge-London: MIT Press. https://mitpress.mit.edu/books/confronting-challenges-participatory-culture.

Jiao, J. L. (2013). The application of and influence of microteaching. Journal of Information and Technology Education of Small and Middle School. <https://www.semanticscholar.org/paper/f9ed1698126ab2a1976d3d0f01f50890f9e7cf22?p2df>.

Karsenti T., Collin S. \& Harper-Merrett T. (2012). Pedagogical -Integration of ICT: Successes and Challenges from 100+ African Schools, Ottawa: ON: IDRC. <https://depot.erudit.org/id/003773dd.

KLER, S. (2015). ICT integration in teaching and learning: Empowerment of education with technology. <https://doi.org/10.15415/iie.2014.22019.

Leal, E. A., Miranda, G. J., \& Casa Nova, S. P. C. (2017). Novas metodologias de ensino aplicadas à contabilidade: existe uma receita? Seção de Pôster apresentado no $11^{\circ}$ Encontro Catarinense dos Coordenadores e Professores de Ciências Contábeis, Florianópolis, SC. learning en vironments. In Jonassen, D. H., e Land, S. M. (Eds.), Theoretical foundations of learning environments. pp. 89-121. Mahwah, NJ: Lawrence Erlbaum.

Leite, Lígia Silva. (2011). Mídia e a perspectiva da tecnologia educacional no processo pedagógico contemporâneo. In: Freire, Wendel (Org.). Tecnologia e educação: as mídias na prática docente. (2a ed.), WAK.

Lope, J. C. G.; Formiga, N. S. (2020). Existe uma personalidade do endividado? Os impactos da impulsividade na atitude ao endividamento em pessoas que têm comprometimento de renda devido ao consumo. Novas Edições Acadêmicas. Beau Bassin.

Lüdke, M; André, M. E. D. A. (2015). Pesquisa em educação: abordagens qualitativas. EPU.

Ma, C. H. (2012). Micro-teaching making learning more interesting and easier. Journal of China Teacher. <http://paper.chinateacher.com.cn/zgjsb /html/2012-10.Out.content_79962.html.

Maia, M. C. O. (2003). Uso da Tecnologia de Informação no Uso da Tecnologia de Informação para a Educação a Dra a Educação a Distância no Ensino Superior. São Paulo, FGV-EAESP, 294f. Tese (Doutorado em Administração de Empresas). FGV-EAESP. Área de concentração: Produção e Sistemas de Informação.

Mendonça, J. R. C. et al. (2013). Competências Eletrônicas de Professores para Educação à distância no Ensino Superior no Brasil: discussão e proposição de modelo de análise. Fórum da Gestão do Ensino Superior nos países e regiões de língua portuguesa, II, b.

Minayo, M. C. S. (Org.). (2001). Pesquisa social: teoria, método e criatividade. Vozes.

Moutinho S. M. H. C. (2000). Desenvolvimento de um método prescritivo para mudança de sistema de indicadores à luz da Teoria das Restrições. Dissertação de Mestrado em Engenharia da Produção, Programa de Pós-graduação em Engenharia da Produção/UFRGS.

Muro, C., Gabriel, M. (2016). Women Engagement in ICT Professions in Tanzania: Exploring Challenges and Opportunities. Women. $<$ https://www.ijcit.com/archives/volume5/issue5/Paper050505.pdf

Muslem, A., Yusuf, Y. Q., \& Juliana, R. (2018) Perceptions and barriers to ICT use among English teachers in Indonesia. Teaching English with Technology, 18(1), 3-23. <http://cejsh.icm.edu.pl/cejsh/element/bwmeta1.element.desklight-34ba375d-50d4-4341-9c9b-6e6fd275c1c0/c/ARTICLE1.pdf.

Nagib, L.; Silva, D. (2020). Adoção de metodologias ativas e sua relação com o ciclo de vida e a qualificação docente no ensino de graduação em ciências contábeis. Revista Contabilidade e Finanças, 31(82), 145-164, 24 jan. <http://www.revistas.usp.br/rcf/article/view/165971.

Oliveira, S. R. de; Batista, S. S. dos S.; \& Almeida, I. B. P. de. (2020). Curricular theories and practices in professional and technological education. Research, Society and Development, 9(1), e167911807. 10.33448/rsd-v9i1.1807. https://rsdjournal.org/index.php/rsd/article/view/1807. 
Research, Society and Development, v. 10, n. 3, e51710313710, 2021

(CC BY 4.0) | ISSN 2525-3409 | DOI: http://dx.doi.org/10.33448/rsd-v10i3.13710

Ojo, O., \& Adu, E. (2018). The effectiveness of information and communication technologies (ICTs) in teaching and learning in high schools in Eastern Cape Province. South African Journal of Education, 38(2), 1-11. <https://doi.org/10.15700/saje.v38ns2a1483.

Oliveira, V. A. (2015). Tecnologias da informação e comunicação: um estudo qualitativo com docentes do Curso de Pedagogia. Dissertação de Mestrado. Programa de Pós -Graduação em Tecnologia, Universidade Tecnológica Federal do Paraná, Curitiba, Paraná, Brasil

Pain, H. (2009). Innovation in qualitative research methodology: annotated bibliography. NCRM Working Paper. Southampton, UK: National Centre for Research Methods.

Pereira, N. V.; \& Araújo, M. S. T. (2020). Use of technological resources in Education: paths and perspectives. Research, Society and Development, 9(8), e447985421, 10.33448/rsd-v9i8.5421. https://rsdjournal.org/index.php/rsd/article/view/5421 .

Pozo, J. (2008). A sociedade de aprendizagem e o desafio de converter informação em conhecimento. Tradução de Antonio Feltrin. Artmed.

Podsakoff, P. Mackenzie, Scott \& Lee. Podsakoff, N. (2003). Common Method Biases in Behavioral Research: A Critical Review of the Literature and Recommended Remedies. Journal of Applied Psychology. https://www.researchgate.net/publication/279796507_Common_Method_Biases_in_Behavior al_Research_A_Critical_Review_of_the_Literature_and_Recommended_Remedies.

Silva, A. M. P. M., \& Coll, C. (2015). Content validity of the questionnaire on learning experiences associated with the use of digital information and communication technologies by undergraduates In: International Conference on Research in Educational and Science, Antalya. International Conference on Research in Educational and Science (ICRES). Proceedings Book. Antalya: International Journal of Research in Education and Science, $179-186$.

Unesco. (2015). Information and communication technology in education. Paris. <http://unesdoc.unesco.org/images/0012/001295/129538e.pdf.

Valente, J. A. (2013). As tecnologias e as verdadeiras inovações na educação. In M. E. Almeida, P. Dias, e B. Silva, Cenários de inovação para a educação na sociedade digital. Edições Loyola, pp. 35-46.

Vieira, R. S. (2011). O Papel das tecnologias da informação e comunicação na educação a distância: um estudo sobre a percepção da educação a distância o professor/tutor. Formoso-Ba: Universidade Federal do Vale do São Francisco (UNIVASF), 10, 66-72.

Vilaça, M. L. C.; \& Araujo, E. V. F. de. (2016). Tecnologia, sociedade e educação na era digital. Duque de Caxias: Unigranrio. <http://www.pgcl.uenf.br/arquivos/tecnologia,sociedadeeeducacaonaeradigital_011120181554.pdf.

Wiebusch, A. \& Lima, V. M. R. (2016). Inovação nas práticas pedagógicas no Ensino Superior: possibilidades para promover o engajamento acadêmico. Revista acadêmica da PUC-RS - Educação por Escrito. http://revistaseletronicas.pucrs.br/ojs/index.php/porescrito/article/view/31607/0.

Willig, C. (2013). Introducing qualitative research in psychology. McGraw-hill education (UK).

Yin, R. K. (2010). Estudo de caso: planejamento e métodos. (4a ed.), Bookman. 